\title{
Moving Target Tracking Based on Cam-shift Algorithm
}

\author{
Jing $\mathrm{Ji}^{\mathrm{a}}$, Jiapeng Tian ${ }^{\mathrm{b}}$ and Yansong Deng ${ }^{\mathrm{c}, *}$ \\ School of Southwest Minzu University, Sichuan 610225, China. \\ ai1996104@163.com, bt49971@163.com, c342547288@qq.com \\ *Corresponding author: Yansong Deng
}

\begin{abstract}
Keywords: Cam-shift, Mean-shift, Raspberry Pi, Image processing.
\end{abstract}
\begin{abstract}
The design of the video transmission system has been applied more and more widely in the daily study and life. In the meantime, implementation and optimization of the system which track moving target's algorithm are also of great significance. In order to adapt the development of daily demand, we build a Wi-Fi hardware platform combined with Raspberry Pi 2B and USB camera in this paper. The OpenCV architecture platform is configured under the Linux system, using $C$ language combined with Cam-shift tracking algorithm to implement real-time moving target tracking. At the same time, Cam-shift algorithm which we use in this paper are both improvement and promotion of the Mean-shift algorithm. Mean-shift is a nonparametric method of the density function gradient estimation, by iterating optimization we can find the probability distribution of extreme value to locate the target. Cam-shift is a continuous adaptive computation of Mean-shift, which can be regarded as one of the best methods that used to track the moving target.
\end{abstract}

\section{Introduction}

With the increase of population, city management problem has become particularly important. Facing the problem of insufficient police force, however, a new type of management style is raging in our cities construction. That is the urban justice system. And this system also gradually become a magic weapon of the city in the process of law enforcement. Comprehensive coverage, and accurate positioning make law enforcement alert can be found in time and act at once. The public gain the maximum protection of people's property security. And when law enforcement use the justice system, they will often use a technique, which is a kind of image processing applications, and also known as moving target tracking. Image tracking can be used not only in the justice system and its application in our daily life is also very wide. In the near future, as long as there is the place of cameras can see the figure of image tracking. Machine was required real-time tracking activities of the human body by identifying the movement of body position. Human body tracking can be used in the video, in order to achieve continuous tracking the position of the human body and direction of movement, finally realizes to follow the human body. When this system is put into use and keep improving and innovation, we can live in a new, safe society.

\section{Cam-shift Algorithm}

\subsection{Two Kind of Tracking Method.}

Usually, we comprehend the movement of objects in two aspects: recognition and modeling. Recognition is to find the objects in the frame of video streaming, which was interested in the frame before. In the process, we can get the preliminary calculation of the object's actual location [1]. The purpose of modeling is to solve the problems above .Mean-shift and Cam-shift are two tracking method. Mean-shift algorithm is a kind of nonparametric method which is to estimate density function gradient, by iterating optimization to find the probability distribution of extreme value to locate the target. And the main idea of the paper is Cam-shift, which is named "continuously the adaptive mean -shift". 


\subsection{Mean-shift Algorithm.}

Cam-shift algorithm is based on the Mean-shift algorithm, in other words, Cam-shift is a improvement of Mean-shift. So at the mean time we us camshaft algorithm, we are supposed to master the principle and implementation process of Mean-shift. Mean-shift is a method that looking for stable and local extremeum between the density distribution of a set of data. Mean-shift algorithm's steps are as follows:

(1)According to the color's probability distribution of the image, select out the search window.

That is, edit and select the initial position of the window, the type of the window, the shape, the size. The types of window including the uniform type, type of polynomial, exponential type and the Gaussian type and so on. While, the shape of the window is likely to be symmetrical, askew, may rotate, circular rectangular and so on.

(2)Calculate the window's center of gravity and adjust the size of the search window.

Mean-shift algorithm's formula can simplify the nuclear of the rectangle.

We can simplify the Mean-shift vector quantity into computing the center of gravity of the image pixels' distribution:

$$
x_{c}=M_{10} / M_{00} \quad y_{c}=M_{01} / M_{00}
$$

The zero moment in the above formula is:

$$
M_{00}=\sum_{x} \sum_{y} I(x, y)
$$

The first moment in the above formula is:

$$
M_{10}=\sum_{x} \sum_{y} x I(x, y) \quad M_{01}=\sum_{x} \sum_{y} y I(x, y)
$$

(3)After have computed the center of gravity in second step, we are supposed to move the center of the search window to the previous center.

(4)If the moving distance is greater than the preset fixed threshold, we can return back to second step. Stop computing till the searching window's moving distance is less than the preset fixed threshold, or the number of loop operation reach the limit.

In general, the Mean-shift algorithm is to state that how to move the center of the searching window to the window's center of gravity. However, this kind of moving will often cause that the searching window's content changed. So, we should repeat calculation the center of the gravity and locate the center of the window.

\subsection{Cam-shift Algorithm.}

What has mentioned above is that camshaft is an improvement of Mean-shift. The differences between Mean-shift algorithm and Cam-shift algorithm is that the size of Cam-shift algorithm's searching window can adjust automatically. That is to say, the Mean-shift algorithm is extended to the continuous image sequence [2]. In simple term, algorithm implementation is to operate all the video frames by Mean-shift algorithm and regard the searching window' size and its center position in previous frame as the initial value of next frame. Iterate again and again, we can track the moving target successfully at last.

Camshaft algorithm steps are as fellows:

(1)Initialize the searching window.

(2)Calculate the color's distribution probability by using back projection.

(3)According to the method described above, run the Mean-shift algorithm. We can obtain a new size and location of the searching window, and then regard these data as initialized data of next frame.

(4)Applying the value in third step to initialized the next video frame's size and location of the searching window, and then jump to run second step repeatedly.

Compared with the Mean-shift algorithm, Cam-shift algorithm that has been optimized can undertake the continuous image sequence, that is to say, Cam-shift can track the moving target through the camera in video. Also the he requirement of system resources is not very stringent. In the case of simple background, we can obtain a good tracking effect. 


\section{The Hardware Platform}

\subsection{Raspberry Pi.}

Raspberry Pi 2B is a micro computer motherboard based on ARM, using SD/Micro-SD card as memory disk. The card board surrounded by four USB 2.0 interface and an Ethernet interface, which can connect the keyboard, mouse and network cable [3]. At the same time, Raspberry Pi have video analog signal output interface and HDMI high definition TV video output interface. Raspberry Pi Foundation provides Debian based on ARM and the Arch Linux distributions for the public to download. And also provides use Python as the main programming language, as well as Java, C programming language etc.

\subsection{System Platform.}

Configuration of software environment: We installed by the official launch of Raspberry Pi Raspbian system, based on Debian system, which aim at the development of Raspberry PI having a strong development. We use the OpenCV3.0 cooperate $\mathrm{C}$ language under Linux kernel system to realize the development, using Gcc compiler together with the Cmake to finish configuration. At the same time in order to facilitate our development and use on the Windows PC, we use the Putty (used to remotely manage Linux systems), Winscp (using SSH SFTP client, can be used to copy with the remote computer file), Vnc (one part is to the client application Vncviewer, the other part is the server application Vncserver) software under Windows environment [4]. Those has be stated above can realize the remotely login operation between different systems, file transfer, the develop of video images, more facilitate in the project of embedded Raspberry PI platform's development.

\section{Algorithm Implementation}

Based on the experimental platform for embedded platform Raspberry PI 2B, it adopts four nuclear architecture Cortex-A7 processor, 1 GB of RAM. Operating system USEs its official launch Raspbian system, using $\mathrm{C}$ language and $\mathrm{C}++$ language programming, camera maximum resolution of $1280 \mathrm{x}$ 720 , to 5 million pixels, the camera target tracking in video detection. Object to be tested as a blue object (The experimental results is shown Figure1 to Figure4), Raspberry Pi 2B to track the target detection [5], using the Putty and VNC remote SSH logging, available through the network PC to land management, achieve the goal of real-time tracking.

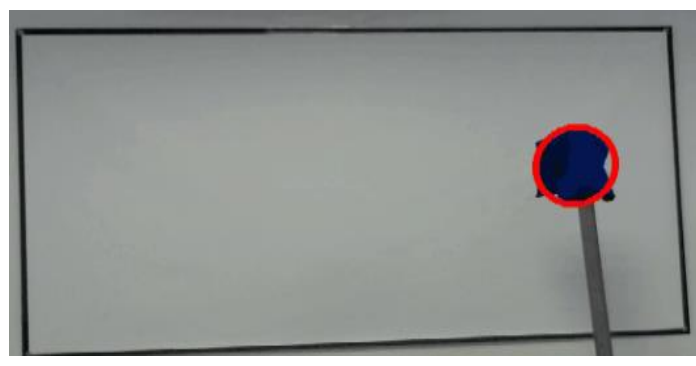

Fig. 1 image of processing

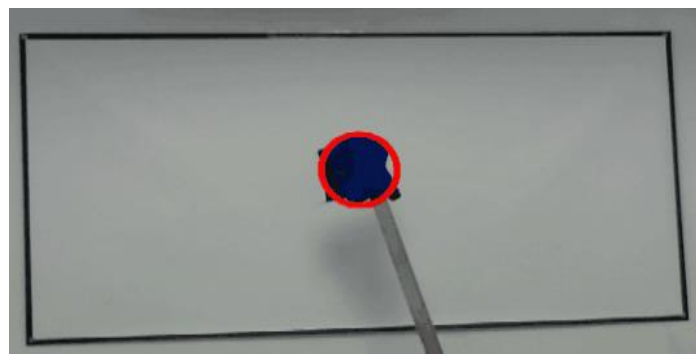

Fig. 2 image of processing 


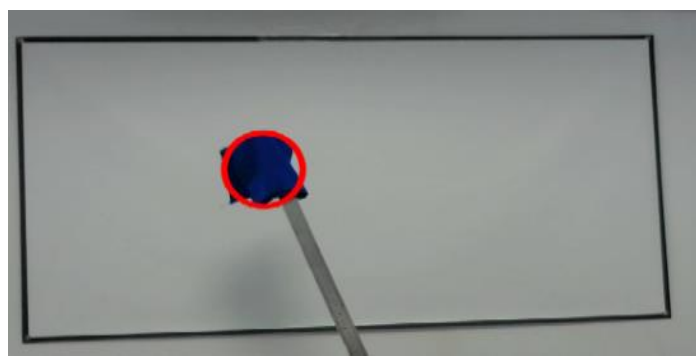

Fig. 3 image of processing

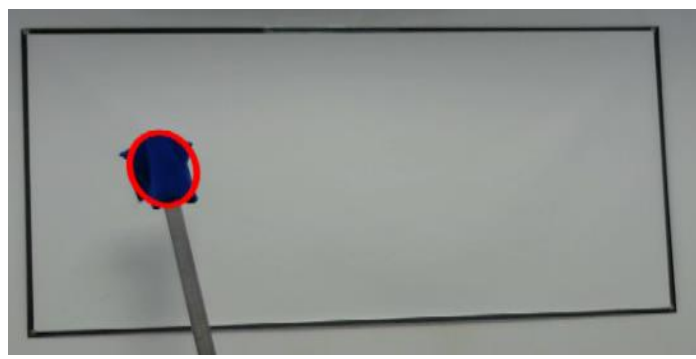

Fig. 4 image of processing

\section{Conclusion}

This paper expounds the software and hardware platform of Cam-shift tracking algorithm, the principle of algorithm and implementation as well as the algorithm verified. But there are still some shortcomings. Tested in the colorful background can cause interference to track target detection and result in the image tracking come to bad effect, the most serious that there will be false positives.

In conclusion, this algorithm platform, though there are some problems, but there are still many advantages on contrast artificial detection. And the algorithm is in the preliminary. This technology is not mature enough and having very big development space. Also need continuous improvement and innovation in later period.

\section{Acknowledgements}

This work was partially supported by National Nature Science Foundation (61673016), SMU Construction Projects for Graduation Degree Programs (2017XWD-S0805), Advance Research Pro-gram of Electronic Science and Technology National Program (2017YYGZS16) and Sichuan Youth Science and technology innovation research team (2017TD0028), also was financially supported by students innovation training project of SMU (201610656036).

\section{References}

[1]. Gary Bradski, Adrian Kaebler. Learing OpenCV. Publishing House of Tsinghua University, Beijing, 2009.

[2]. Robert Laganiere. OpenCV computer vision application programming cookbook. Publishing House of People's posts And Telecommunications, BeiJing,2015.

[3]. Peter Memberry, David Hows. Based on Linux. Publishing House of People's posts And Telecommunications, BeiJing,2014.

[4]. Rick Golden, et al. Raspberry Pi networking cookbook. Science Press, Beijing, 2014.

[5]. Ruth Suehle, Tom Callaway, et al. Raspberry Pi hacks. Publishing House of People's posts And Telecommunications, BeiJing,2015. 